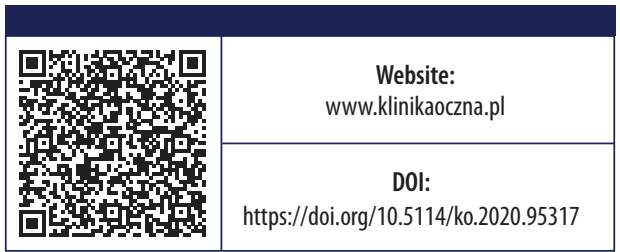

\title{
Ophthalmic manifestation of Creutzfeldt-Jakob disease in the Heidenhain variant - a case report
}

\author{
Anna Linke', Sławomir Cisiecki ${ }^{1,2}$, Karolina Bonińska ${ }^{1,2}$, Maciej Bednarski ${ }^{1,2}$ \\ 'Ophthalmology Department, Dr K. Jonscher Municipal Medical Centre, Lodz, Poland \\ 2Julianów Medical Centre, Lodz, Poland
}

\begin{abstract}
We present the case of a 59-year-old female patient with the sporadic form of Creutzfeldt-Jakob disease in the Heidenhain variant. The main ocular symptoms were initial bilateral deteriorated visual acuity and final blindness. Due to a very low prevalence, non-specific symptoms and rapid course, making a quick and ac-
\end{abstract}

curate diagnosis can be challenging. Vision impairment may occur in about $10 \%$ of patients with a sporadic form of Creutzfeldt-Jakob disease at the time of diagnosis and 50\% during the disease. So far, no effective treatment has been found. The death of our patient occurred 9 weeks after the first symptoms.

KEY WORDS: Creutzfeldt-Jakob disease, Heidenhain disease, prions.

\section{INTRODUCTION}

Creutzfeldt-Jakob disease (CJD) is a non-curable neurodegenerative prion disease, also known as transmissible spongiform encephalopathy (TSE). Its incidence is estimated at $1-1.6: 1,000,000$. The disease involves deposition of proteins demonstrating an abnormal conformation, called prion proteins (PrPs), in the central nervous system and in peripheral tissues. These proteins are formed as a result of conversion of the correct cellular form $\operatorname{PrP}^{\mathrm{C}}$ into the pathogenic one $\operatorname{PrP}^{\mathrm{Sc}}$, showing a different secondary structure $[1,2]$.

There are four forms of the disease that differ in the etiological factor. They are:

1. The sporadic form (sCJD), constituting approx. $85 \%$ of cases - with unknown etiology and a very rapid course, developing as a result of a spontaneous change of conformation of the prion protein.

2. The familial form (fCJD), constituting approx. 15\% of cases - hereditary, associated with presence of mutation of the PRNP gene, encoding the prion protein.

3. The iatrogenic form (iCJD), constituting less than $1 \%$ of cases - caused by infection during surgical procedures.

4. Variant CJD (vCJD), occurring in less than $1 \%$ of patients - most commonly affecting young people, below the age of 30 years. Duration of the disease is usually longer than in other forms. It is associated with consumption of food produced of cattle affected by bovine spongiform encephalopathy (BSE) [3, 4].
Creutzfeldt-Jakob disease incubation time ranged from several months to over ten years [5].

The current clinical criteria of SCJD define the possibility of making a diagnosis as possible, probable and certain [6]. The final diagnosis may be made only based on postmortem neuropathological examination, or on examination of a biopsy specimen. Imaging techniques are helpful in in vivo diagnosis: electroencephalography (EEG) and magnetic resonance imaging (MRI) of the head, as well as laboratory tests for the presence of the 14-3-3 protein and the tau protein in the cerebrospinal fluid [7]. The most specific (98\%) way to detect pathological prion protein is RT-QuIC (real-time quaking-induced conversion) [8].

The first symptoms may be fatigue, dizziness, mental disorders, memory impairment, and vision impairment. Over time, gait disturbances, aphasia, ataxia, akinetic mutism, dystonia, and features of pyramidal and extrapyramidal system injury may also appear.

One of the clinical presentations of sCJD is the so-called Heidenhain syndrome, which is characterized by isolated visual symptoms and occurs in $4-20 \%$ of sCJD cases. The peak incidence is observed in patients aged 50-88 years [9]. Symptoms include deterioration of visual acuity, visual hallucinations, depth perception disorders, visual perseverance, tunnel vision, diplopia, or cortical blindness. Heidenhain syndrome is associated with the average survival time shorter by about 2 months. Death usually occurs within a year of 
the first symptoms of CJD. No effective treatment has been developed so far.

\section{CASE REPORT}

We present a case of a 59-year old female patient, admitted to the Neurology Ward in September, last year.

General examination. At admission the patient reported deterioration of visual acuity, speech disorders (slurred, confused speech), vertigo and balance disorders. These symptoms had been intensifying over previous 3 weeks. At admission the patient was in rather good condition, in full logical verbal contact, oriented in place, time and herself. Computed tomography of the head done without contrast enhancement showed no departures from the normal.

Neurological examination. During the hospitalization fresh memory disorders, psychomotor sluggishness, dysarthria, ataxia of upper and lower extremities, bilateral positive kinn reflex and wobbly Romberg's test were observed.

Ophthalmological examination. An ophthalmologic consultation was conducted. The following medical history was considered: a procedure of buckling for retinal detachment in 2015, surgical removal of cataract from the right eye in 2017, and YAG-capsulotomy two years later. The patient was regularly ophthalmologically monitored because of high myopia (-10 Dsph) and confirmed Fuchs dystrophy. The following visual acuity was noted during the last visit, in $\mathrm{Au}-$ gust last year: $\mathrm{V} \mathrm{RE}=5 / 25 \mathrm{cc}-10 \mathrm{Dsph}$ and V LE $=5 / 32 \mathrm{cc}$ -10 Dsph. Considering progressive Fuchs dystrophy, the patient was recommended to use drops with $60 \%$ glycerol solution, and a proposition was made to qualify her for transplantation of cornea (finally the procedure was not performed).

On the consultation day the patient's visual acuity in both eyes was hand movement in front of eyes. IOP measured in both eyes with Goldmann applanation tonometry was $17 \mathrm{mmHg}$. A full ophthalmological examination was performed with indirect biomicroscopy after dilating the pupils with $1 \%$ tropicamide solution. In the right eye the following departures from the normal were found: the reduced translucency of the cornea, its paracentral temporal part is edematous (Figure1A); choroid-retinal atrophy around the optic disc; circumferential intussusception bank corresponding to buckling. In the left eye: a minor central edema of the cornea, cornea with mildly reduced translucency (Figure 1B). The lens with clouding in cortical-medullary layers and in the subcapsular posterior layer; choroid-retinal atrophy around the optic disc.

Swept-Source-OCT. In the Swept-Source-OCT image of the left eye: contour of the fovea is correct. Reduced thickness of the retina with thinning of the layer of the pigmented epithelium (Figure 2).

Due to the lack of fixation, the Swept-Source-OCT examination of the right eye was not possible.

Ultrasonography. The US examination was performed: unremarkable (Figure 3).

The examination indicated absence of correlation between vision deterioration and the ophthalmological condition. Suspicion of central origin of vision disorders was made.

Neurological imaging. Further imaging diagnostics was performed during hospitalization in the Neurology Ward. In the EEG, dominant triple-phase sharp waves, frequency of $3 \mathrm{~Hz}$ and a higher amplitude in frontal leads up to $150 \mathrm{uV}$ with antero-posterior delay within $100 \mathrm{~ms}$ were observed (Figure 4).

MRI of the head showed signs of injury of basal ganglia. In both nuclei caudati and - to lesser extent - in lentiformis nuclei, there is a high signal visible bilaterally in T1 and T2-weighted images, and in the FLAIR sequence, as well as in DWI with no contrast enhancement (Figure 5A-D).

Five days later EEG was repeated, which registered periodic discharges of sharp-wave complexes (PSWCs) every 1-1.5 s in right-side leads. The basic activity was composed of waves of $6 \mathrm{~Hz}$ frequency (Figure 6).

Basing on all those reports, a suspicion of CJD was made.

Laboratory tests. Lumbar puncture was performed to collect cerebrospinal fluid. The protein 14-3-3 was found. The overall clinical presentation and results of additional investi-
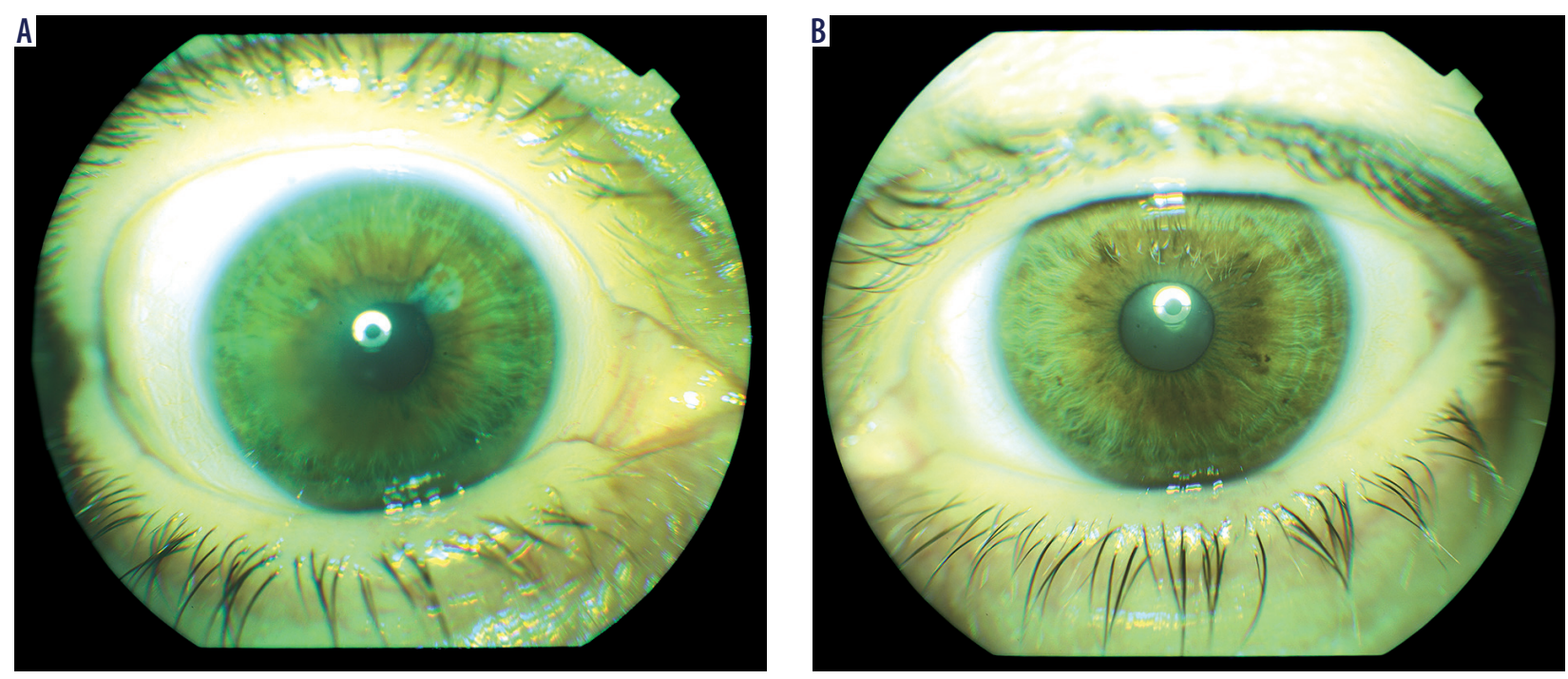

Figure 1. Anterior segment photography of the right (A) and left (B) eye of the patient with noticeable Fuchs' dystrophy 


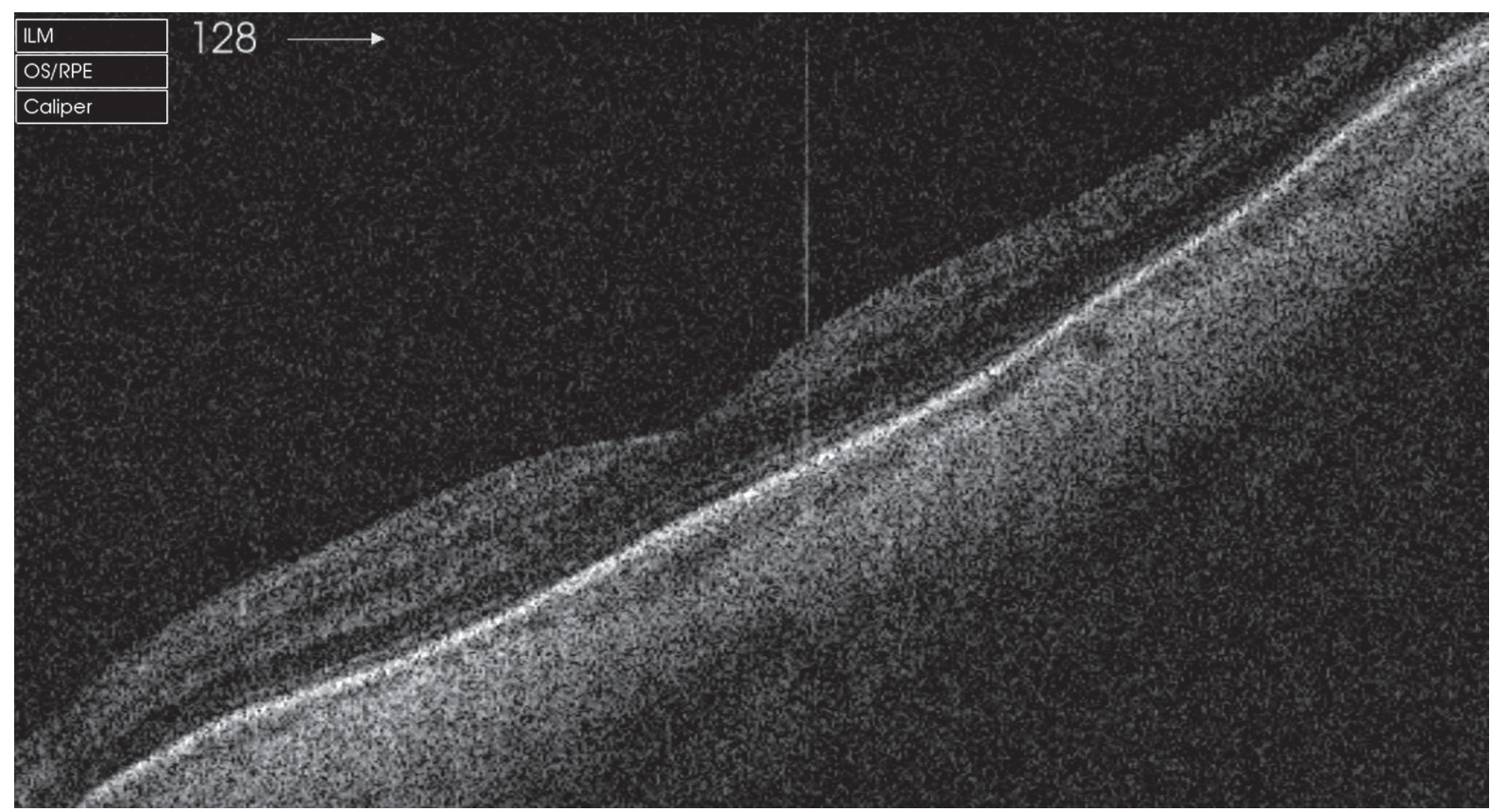

Figure 2. The Swept-Source-OCT scan showing retinal thinning with retinal pigment epithelium atrophy at the macula

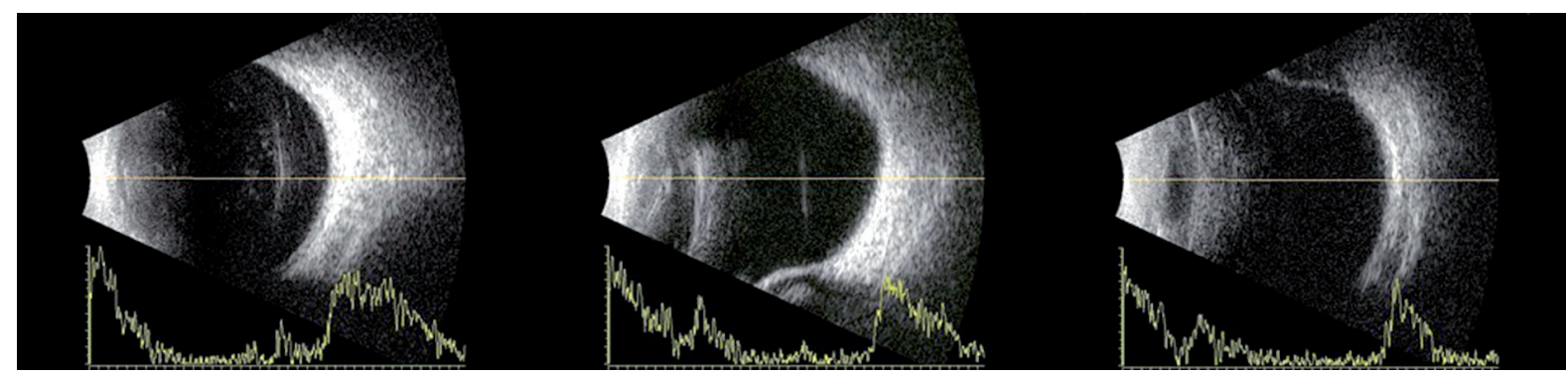

Figure 3. The B-scan images show circumferential scleral buckle and no other evident abnormalities

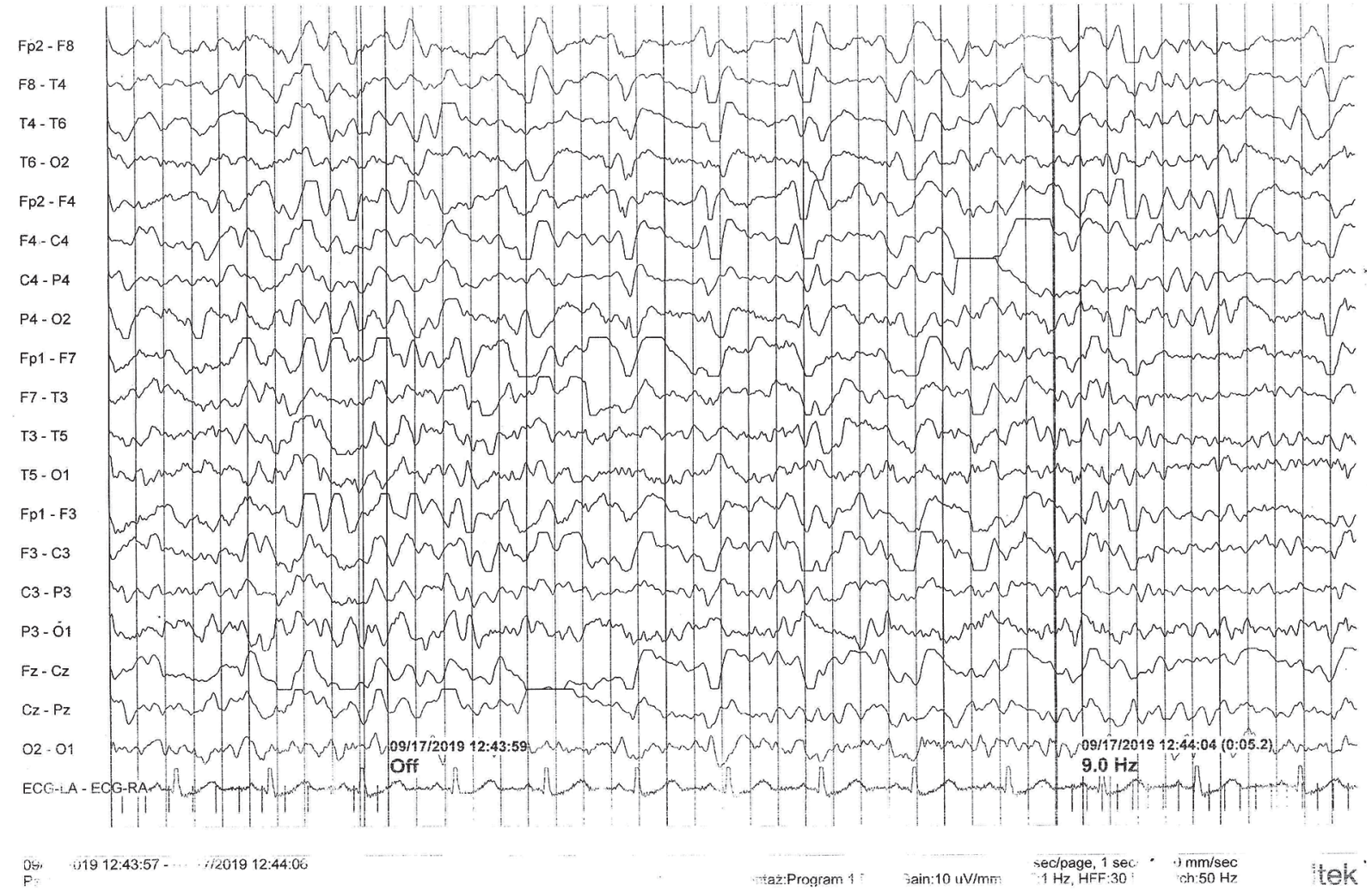

Figure 4. Results of EEG recordings with predominant sharp-wave complexes, characteristic for toxic-metabolic encephalopathy 

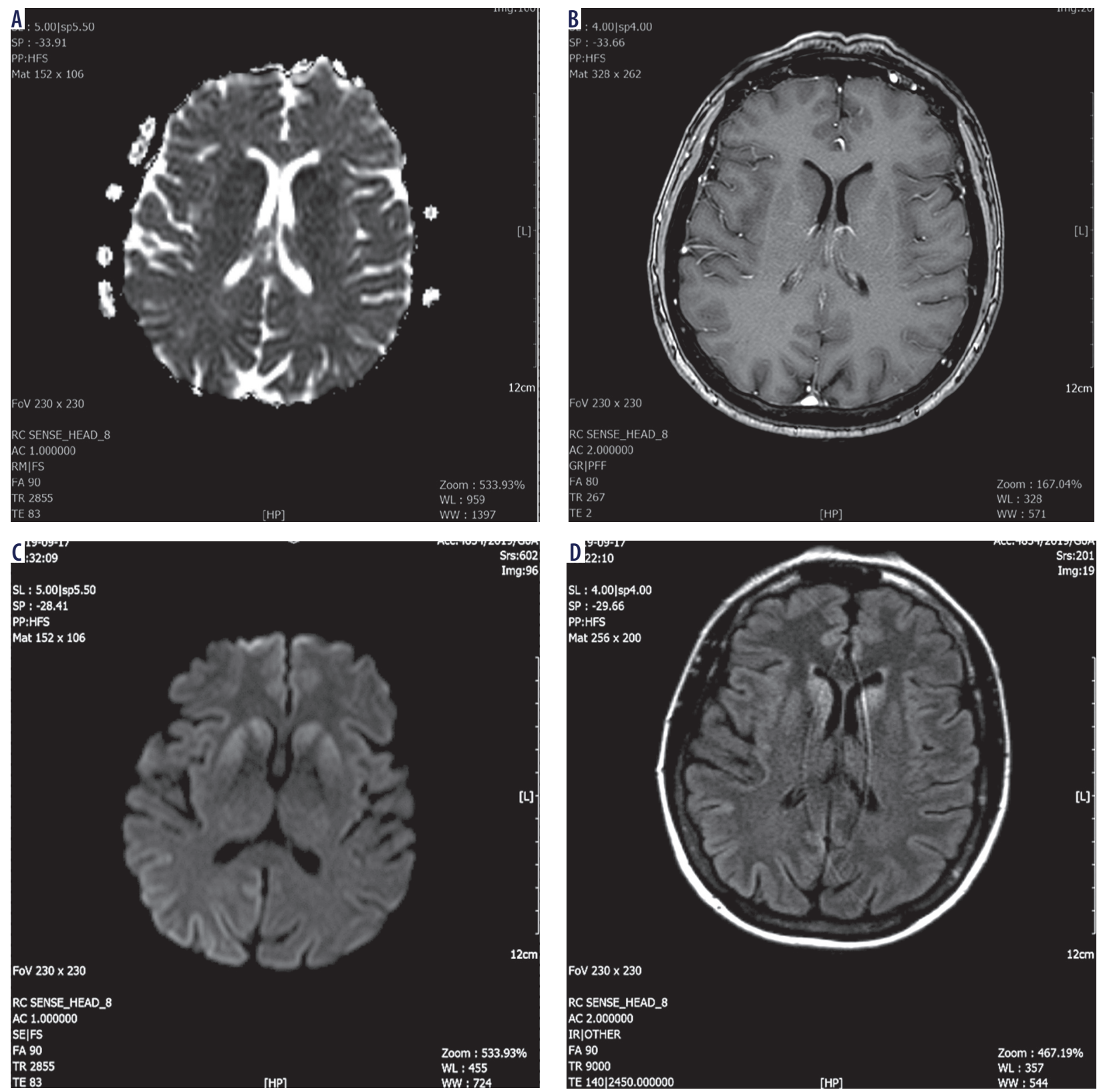

Figure 5. Cerebral MRI scans of a patient with sporadic form of Creutzfeld-Jakob disease in ADC maps (A), T1 images (B), DWI maps (C), FLAIR sequences (D). Symetric high signal abnormalities of basal nuclei

gations, according to diagnostic criteria, indicated a probable diagnosis of CJD.

After two weeks of hospitalization, the patient was discharged in an intermediate general condition with recommendations for further care in a neurological outpatient clinic and she was referred to the palliative medicine ward. Three days later the patient was re-admitted to the Neurology Ward because of deteriorated verbal logical contact, swallowing disorders and ataxia. The examination demonstrated: dementia, amblyopia, akinetic mutism, dysphagia, quadriplegic ataxia, bilateral kinn reflex, as well as blindness. A probe was installed, but the patient removed it on the next day. During the hospitalization the logical and verbal contact with the patient was gradually deteriorating, up to the level of a complete loss of logical contact, speaking single words, sounds, signs of extrapyramidal syndrome. After 10 more days the patient in overall intermediate condition was discharged from the hospital, and transferred to the palliative medicine ward. Two weeks later the patient died.

\section{DISCUSSION}

We present a case of a female patient with a sporadic form of CJD, with cortical blindness. This condition was first described in 1929 by the German neurologist Adolf Heidenhain. Due to the very low morbidity rate, non-specific symptoms and rapid course, making a quick and accurate diagnosis may be challenging for the ophthalmologist. Although there may be various non-specific ophthalmic symptoms, most often there is a significant sudden deterioration of vision. In the absence of accompanying tangible deviations from the normal in ophthalmic examination, as well as rapid progression of the disease, the ophthalmologist should suspect the central origin 


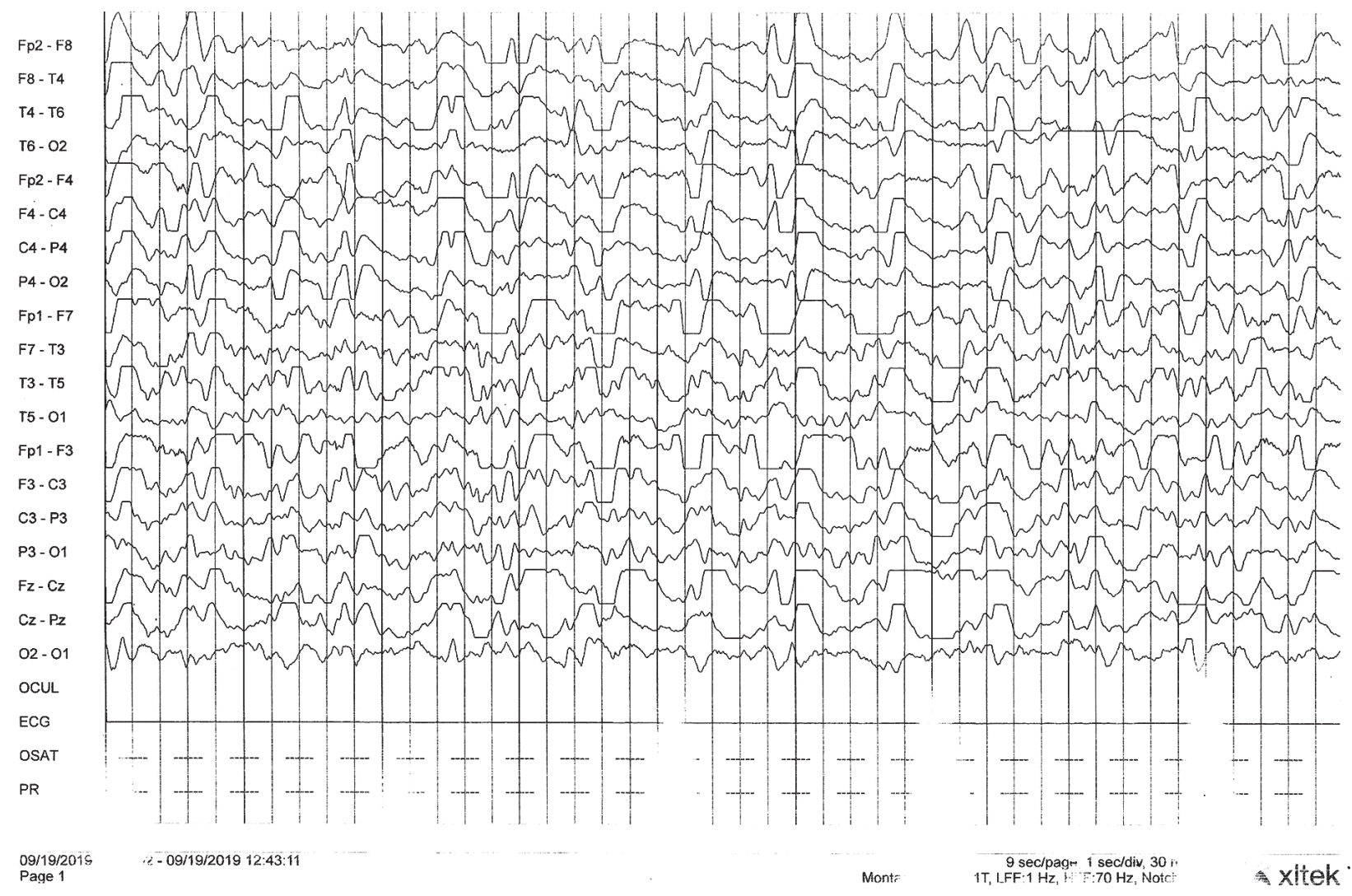

Figure 6. Follow up EEG imaging with persistent periodic sharp-wave complexes (PSWCs)

of symptoms reported by the patient. Cooperation with a neurologist, and sometimes also with a psychiatrist is necessary.

Differential diagnosis should consider other neurodegenerative disorders, including Alzheimer's disease, Pick's disease, corticobasal degeneration, progressive supranuclear palsy, dementia with Lewy bodies, as well as vascular, iatrogenic, autoimmune, neoplastic, metabolic and infective causes. Cognitive impairment is characteristic for all those conditions. Additional investigations are necessary.

According to World Health Organization (WHO) criteria, in vivo diagnosis of CJD is based on the progressive dementia symptoms along with atypical visual symptoms, supplemented with neuroimaging [12]. MRI of the head may be initially unremarkable, but with time characteristic hyperintensive symptoms in sequences DWI and FLAIR become manifested. Periodic sharp-wave complexes in EEG or confirmed presence of the protein 14-3-3 and the protein tau in the cerebrospinal fluid may suggest the diagnosis, but are not pathognomonic for sCJD. The EEG record with PSWCs may be also registered in the Alzheimer's disease and Lewy dementia. Moreover, PSWCs may appear at any stage of disease, and may not necessarily be present from the very beginning of symptoms. Post-mortem examination is the sole source of the certain final diagnosis.

The patient described by us met the diagnostic criteria of probable sCJD (neurological symptoms, presence of the protein 14-3-3 in the cerebrospinal fluid, triple-phase sharp wave complexes present in the EEG from the beginning and duration of symptoms less than two years). The results of laboratory investigations excluded presence of an infectious factor. Tumor markers were also negative.

Etiology of the sporadic form of CJD remains unknown. Despite the progressive Fuchs dystrophy, the patient did not receive corneal transplant surgery, which excludes the iatrogenic aspect. However, the possibility of prion transmission as a result of transplantation of infected tissue, as well as through the surgical instruments themselves should be considered.

Orrù et al. described eleven patients who had received corneal transplant from donors with sCJD [13]. Post-mortem immunohistochemical assays demonstrated accumulation of prions in the choroid, sclera, cornea, optic nerve, vitreous body, lens, extraocular muscles and retina - both inner and outer plexus layers - from donors. Surprisingly, the highest level of prions was not found in the transplanted cornea, but in the optic nerve and retina in all patients studied. This is particularly important due to the possibility of post-mortem examination of tissues for infection, in order to qualify the donor for later transplantation.

From the first hospitalization the presented patient lived for only 6 weeks. This confirms the extremely aggressive course of the Heidenhein form. According to literature data, the survival time does not depend on the age at which the disease is diagnosed $[14,15]$. It is on average 5.7 months in Heidenhain disease and 7.5 months in the sporadic form. 
There is no effective treatment so far. Not many clinical studies on this subject were also conducted.

\section{CONCLUSIONS}

In patients with an ocular disorder of unknown cause, when excluding an ophthalmic background, sporadic CJD should be considered in the differential diagnosis. Besides the clinical presentation, main roles in the diagnosis are played by MRI, EEG and presence of the protein 14-3-3 in the cerebrospinal fluid.

\section{DISCLOSURE}

The authors declare no conflict of interest.

\section{References}

1. Prusiner SB. Novel proteinaceous infectious particles cause scrapie. Science 1982; 216: 136-144.

2. Heinemann U, Krasnianski A, Meissner B, et al. Creutzfeldt-Jakob disease in Germany: a prospective 12-year surveillance. Brain 2007; 130: 1350-1359.

3. Ladogana A, Puopolo M, Croes EA, et al. Mortality from Creutzfeldt-Jakob disease and related disorders in Europe, Australia and Canada. Neurology 2005; 64: 1586-1591.

4. Takada LT, Geschwind MD. Prion diseases. Semin Neurol 2013; 33: 348-356.

5. Brown P, Brandel JP, Preece M, Sato T. latrogenic Creutzfeldt-Jakob disease: the waning of an era. Neurology 2006; 67: 389-393.

6. National Creutzfeldt-Jakob Disease Surveillance Diagnostic Criteria. National Creutzfeldt-Jakob Disease Surveillance 2010. http://www.cjd.ed.ac.uk

7. Ciepierski WM, Adamczyk-Sowa M, Męcik Kronenberg T, Wierzcbicki K. Diagnostics of sporadic Creutzfeldt-Jakob disease - literature review. Wiad Lek 2019; 72: 1995-2004.

8. Atarashi R, Satoh K, Sano K, et al. Ultrasensitive human prion detection in cerebrospinal fluid by real-time quaking-induced conversion. Nat Med 2011; 17: 175-178.

9. Cooper SA, Murray KL, Heath CA, et al. Isolated visual symptoms at onset in sporadic Creutzfeldt-Jakob disease: the clinical phenotype of the "Heidenhain variant". Br J Ophthalmol 2005; 89: 1341-1342.

10. Baiardi S, Capellari S, Ladogana A, et al. Revisiting the Heidenhain variant of Creutzfeldt-Jakob disease: evidence for prion type variability influencing clinical course and laboratory findings. J Alzheimers Dis 2015; 50: 465-476.

11. Kropp S, Schulz-Schaeffer WJ, Finkenstaedt M, et al. The Heidenhain variant of Creutzfeldt-Jakob disease. Arch Neurol 1999; 56: 55-61.

12. Report of a WHO consultation on global surveillance, diagnosis and therapy of human transmissible spongiform encephalopathies. Geneva, Switzerland, 9-11 February 1998. WHO/EMC/ ZDI/98.9. WHO Weekly Epidemiological Record 1998; 73: 361-372.

13. Orru CD, Soldau K, Cordano C, et al. Prion seeds distribute throughout the eyes of sporadic Creutzfeldt-Jakob disease patients. mBio 2018;9.

14. Tanev KS, Yilma M. An unusually presenting case of SCJD - the VV1 subtype1. Clin Neurol Neurosurg 2009; 111: 282-291.

15. Lin CJ, Chen L, Chen P, Chen SJ. A case of sporadic Creutzfeldt-Jacob disease with unusually long survival: correlation with clinical features, electroencephalogram, and brain magnetic resonance imaging. Int J Gerontol 2012; 6: 292-294. 